\title{
ANALYSis OF Watermarking TechniQues For Medical Images Preserving RoI
}

\author{
Sonika C. Rathi ${ }^{1}$ and Vandana S. Inamdar ${ }^{2}$ \\ ${ }^{1}$ Department of Computer Engineering, College of Engineering Pune, \\ Shivajinagar, Pune University, Maharashtra, India \\ sonikacrathi@gmail.com \\ ${ }^{2}$ Department of Computer Engineering, College of Engineering Pune, \\ Shivajinagar, Pune University, Maharashtra, India \\ vhj.comp@coep.ac.in
}

\begin{abstract}
Telemedicine is a well-known application, where enormous amount of medical data need to be securely transfer over the public network and manipulate effectively. Medical image watermarking is an appropriate method used for enhancing security and authentication of medical data, which is crucial and used for further diagnosis and reference. This paper discusses the available medical image watermarking methods for protecting and authenticating medical data. The paper focuses on algorithms for application of watermarking technique on Region of Non Interest (RONI) of the medical image preserving Region of Interest (ROI). The medical images can be transferred securely by embedding watermarks in RONI allowing verification of the legitimate changes at the receiving end without affecting $R O I$.
\end{abstract}

\section{KEYWORDS}

ROI \& RONI, Segmentation, Authentication, security, medical confidentiality

\section{INTRODUCTION}

Speedy development of internet in every field leads to availability of digital data to the public. Internet has been spread in many applications like telemedicine, online-banking, teleshopping etc. One of this application telemedicine is crucial one, where internet is used to transfer or receive medical data by healthcare professional. Due to advancement in information and communication technologies, a new context of easier access, manipulation, and distribution of this digital data have been established [1]. The medical images can be readily shared via computer networks and easily used, processed, and transmitted by using great spread network [2, 3].

In the last decades, uses of advanced electronic and digital equipments in health care services are increased. In fact, in most of the hospitals physicians diagnose their patients by relying on the provided electronic and digital data (such as Ultrasonic, Computed Tomography (CT), Magnetic Resonance Imaging (MRI) and X-ray images). This results in generation of large number of electro digital data (i.e. medical images) continuously at various health care centers and hospitals around the world.

David C. Wyld, et al. (Eds): CCSEA, SEA, CLOUD, DKMP, CS \& IT 05, pp. 297-308, 2012.

(C) CS \& IT-CSCP 2012

DOI : $10.5121 /$ csit. 2012.2230 
In number of medical applications, special safety and confidentiality is required for medical images, because critical judgment is done on medical images, which leads to the proper treatment. Therefore, it must not be changed in an illegitimate way; otherwise, an undesirable outcome may results due to loss of decisive information. Therefore, there is a need to provide a strict security in medical images to ensure only occurrence of legitimate changes. Now-a-days exchange of medical images between hospitals located in different geographical location is very common. Moreover, as this exchange of "medical reference data" done via unsecured open networks leads to the condition of changes to occur in medical images and creates a threat which results in undesirable outcome. Considering this fact, demand of security is getting higher due to easy reproduction of digitally created medical images.

For copyright protection and authentication of these medical images, digital watermarking is an emerging technique, which includes the embedding and extraction process. In embedding process some secret information is embed in to medical images. Extraction process deals with the extraction of secret message, which embedded in the medical image. If failure occurs in extraction process the physician would come to know that there has been some kind of tampering with that image, and he would take precaution of not making diagnosis based on that image. However, if the extraction process extracts the correct watermark, which generally consumes a few seconds, physician can continue with diagnosis.

Medical images hold decisive property and are very crucial and important part of medical information. Such part of the medical image is called as Region of Interest (ROI). The ROI is helpful in providing further diagnosis by the physician. A small bit of distortion in ROI may lead to undesirable treatment for patient. For securing medical images through watermarking ROI should be preserved and the watermarks can be applied on the remaining part of the image called as Region of Non Interest (RONI). Therefore, application of watermarking in medical images can be considered as two-step process which includes:

1. Extracting ROI form the medical images

2. Applying watermarking on RONI

Different algorithms are available for segmentation of ROI on the different types of medical images. Additionally, there are different algorithms available for applying watermarking.

\section{REGION OF INTEREST (ROI) SEGMENTATION}

Segmentation plays an important role in medical image processing [4, 5]. In medical image analysis segmentation is the first step to be followed, to avoid distortion of ROI [4, 6]. Image segmentation deals with the process of partitioning an image into different regions by grouping together neighborhood pixels based on some predefined similarity criterion [7]. This similarity criterion can be defined by specific properties of pixels in the image. Segmentation in medical imaging is used for the extracting the features, image display and for the measurement of image. The goal of segmentation is to divide entire medical image in to sub regions i.e. (white and gray matter). In addition, this helps in classifying image pixels in to anatomical regions (such as bones, muscles and blood vessels).

Defining the borders of ROI in medical image can simplify the procedure of segmentation. In addition, the step of defining borders of ROI is a crucial one, which helps in determining the result of the application as entire analysis fully relies on the output from segmentation step. There are different approaches (for segmenting the image) defined for the different imaging technologies such as CT, MRI, US, colonoscopy etc. Segmentation is semi-automatic procedure and we need to define a seed point in an image. Therefore, the algorithm, which gives perfect 
result for one application, might not even work for another. Figure 1 shows the ROI part of medical image, where physician performs the diagnosis.

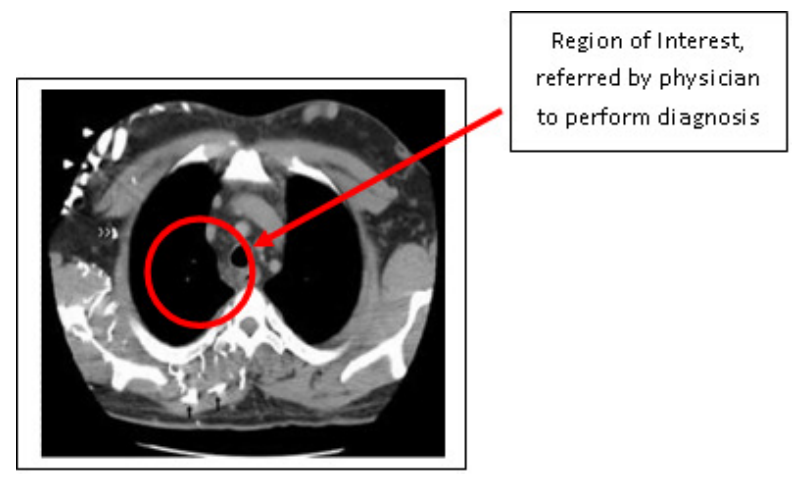

Fig.1: Medical image indicating ROI

We have various existing medical imaging like Computed Tomography (CT), Magnetic Resonance Imaging (MRI), Ultrasound (US), and Positron Emission Tomography (PET) etc. Here, two most common imaging i.e. MRI and CT scan are discussed in detail with their proposed algorithms.

\subsection{Magnetic Resonance Imaging (MRI)}

Magnetic Resonance Imaging (MRI) provides a wealth of information, which is useful for medical examination. In many applications where MRI is used, segmentation of image into different intensity classes are needed, which is regarded as the best available representation for biological tissues $[8,9,10]$. Segmentation plays very important role in MRI process for deciding the spatial location, selecting the operation path, shape, and size of the focus etc. In segmenting MRI images, main requirement is to care about three problems: noise, partial volume effects (where more than one tissue is inside a pixel volume), and intensity in-homogeneity [8, 11]. Due to irregularities of the scanner magnetic fields-static (BO), radio frequency (B1), and gradient fields, intensity in-homogeneities are caused. These irregularities results in producing spatial changes in static tissues of MRI data. When multiple tissues contribute to a single voxel, by making the distinction between tissues along boundaries more difficult leads to the problem of partial volume effects. Adding noise in MRI images can encourage disconnection between segmentation regions. Therefore, for doing segmentation of MRI data on these three difficulties need to focus. There are four different approaches for doing image segmentation: thresholding, clustering, edge detection, and region extraction. This section covers various available MRI segmentation algorithms based on following approaches:

- Thresholding approach

- Clustering approach

- Edge detection approach

\subsubsection{Thresholding}

Thresholding is one of the easiest and most frequently used techniques to segment MRI data by separating the foreground from background of image $[12,13]$. Thresholding approach can further be classified as global thresholding and local (adaptive) thresholding. In global thresholding method, image segmentation is done by providing single threshold value in the whole image 
whereas, in local thresholding, threshold value is assigned to each pixel of image by using local information around the pixel, and then to determine whether the particular pixel belongs to foreground or background these threshold values are used. Due to simplicity and easy implementation of global thresholding, this method is more popular.

P-tile method is one of the earliest thresholding methods based on the gray level histogram [12, 14]. Here P refers to the word "percentile". This algorithm stands on the statement 'Objects in the image are brighter than the background, which occupy a fixed percentage of the picture area'. In this algorithm, threshold is defined as the gray level that mostly corresponds to mapping at least $\mathrm{P} \%$ of the gray level into the object. The experimental results of this method specify that it is suitable for all size of objects, and it provides good anti-noise capabilities. However, this method is not applicable in application where object area ratio is unknown or varies from image to image.

\subsubsection{Clustering}

The goal of clustering approach is to group similar objects and separates the dissimilar objects. That is depending on some perceived similarities this grouping of pixels is done. These clusters then lead in providing natural partitions of pixels that corresponds to the different regions in an image. Conventional clustering algorithms require a prior knowledge regarding the number of clusters, clustering criteria, and nature of data, etc.

There are many algorithms defined for the clustering, such as K-means clustering, Fuzzy c-means (FCM) clustering, possibilistic c-means, possibilistic-fuzzy clustering, intuitive fuzzy c-means (IFCM), and so on. The objective of clustering, for a given set of unlabeled $\mathrm{N}$ samples or data i.e. $\mathrm{X}=\{\mathrm{x} 1, \mathrm{x} 2, \ldots, \mathrm{xn}\}$ is to assign a class label among $\mathrm{C}$ labels to each of $\mathrm{N}$ samples. This number of labels $\mathrm{C}$, considered as the number of regions or number of groups. One of the most widely used clustering algorithms is Fuzzy c- means (FCM) $[15,16]$. In the FCM algorithm, it assigns labels to the data, which are inversely related to relative distance of to the point prototypes that are cluster centers in FCM model. In FCM, proximity of each data or samples, xk, to the center of cluster, vi, is defined as membership or label (uki) of data xk to the ith cluster of $\mathrm{X}$ with following conditions:

$0 \leq u_{k i} \leq 1$ and $\sum_{i=1}^{c} u_{k i}-1, \forall k$

$U=\left[u_{k i}\right]_{N Y C}$ and $V=\left\{v_{1}, v_{2}, \ldots, v_{3}\right]$

FCM algorithm is acknowledged as one of the best clustering algorithm as it resolves various problems, [15, 17], although it still suffers from undesirable solutions with outliers data $[15,18]$. As in FCM algorithm, it requires to provide the exact number of clusters in advance as a prior knowledge. The exact estimation of number of clusters in MRI images, (used in particular for diseased cases), may not be possible to have in advance. To overcome this problem, Krishnapuram and Keller proposed a clustering model named possibilistic c-means (PCM) [15, 18]. In the PCM model the condition of FCM model,

$\sum_{i=1}^{c} \mathrm{u}_{\mathrm{ki}}=1, \forall k$, is relaxed by introducing the new condition as

$\sum_{i=1}^{C} \mathrm{u}_{\mathrm{ki}}=\mathrm{C}, \forall \mathrm{k}$

By providing new condition, PCM model improves its performance over the FCM algorithm, as PCM overcomes the drawback of FCM. PCM is ISODATA based algorithm, which makes use of 
user defined criteria for merging and splitting clusters to discover the number of natural clusters in the data. However, it is very difficult to define these splitting and merging criteria that can be applied on various MR data based on prior assumptions of intensity distribution. Hence, PCM model is very sensitive to initialization and need of additional parameters [15, 19]. Afterward a possibilistic-fuzzy clustering (PFCM) model is proposed. In PFCM algorithm both FCM and PCM model combined by introducing two new parameters $a$ and $b$, where $a$ and $b$ are the weighting factors of FCM and PCM, respectively, to resolve the outlier problems of FCM and sensitivity problem. Again, in PFCM algorithm as it requires selecting additional parameters and extra computation complexity, a new model intuitive fuzzy c-means (IFCM) model is proposed by Dong-Chul Park for MRI image segmentation. The basic operation of IFCM model is same as in FCM except the membership assignment condition. To deal with the problems of membership assignment to noise data IFCM algorithm has been developed. In IFCM, a new measurement called intuition level is introduced by using membership values of FCM and PFCM, $\mathrm{u}_{\mathrm{ki}}$ so that the intuition levels may alleviate the effect of noise data.

\subsubsection{Edge Detection}

For doing the segmentation by using edge detection approach, first step is to extracts the features by obtaining the information from images. Edge detection is a fundamental tool used in most image processing application. It is the process of detecting boundaries between objects and the background in the image, at which the image brightness changes sharply. There are many algorithms to perform edge detection, and all of them classified into two categories Gradient and Laplacian.

Edge detection based on Gradient method initially calculates first derivative of image, and then find its corresponding local maxima and minima values to detect the edges. While, in the Laplacian method after obtaining the second derivative of the image it looks for zero crossing. There are various operators defined i.e. Roberts [20], Prewitt [21], Sobel [22], Canny [23] edge operators to perform Gradient method. These operators include a small kernel rolled up together with the image, which helps in estimating first order directional derivative of the image brightness distribution. This kernel finds edge strength in the direction, which are orthogonal to each other, usually vertically and horizontally. The total value of the edge strength is then obtained by the combination of both the components. Here by creating a matrix centered on each pixel it calculates the edge value. Moreover, if the calculated value is larger than provided threshold, then that pixel is classified as an edge.

With reference to the earlier work of the Marr and Hilderth [21], John F. Canny [23] has developed an edge detection operator named as 'Canny edge detection operator' in 1986. This operator helps in detecting wide range of edged in images by using a multi-stage algorithm. He provided gradient-based-finding algorithm called as optimal edge detector, which becomes most popular and commonly used edge detectors to have the segmented image. Canny edge detector used a method called "Hysteresis", which is proposed to tracing the unsuppressed pixels. Here it uses two threshold values i.e. high and low. After finding the gradient values, algorithm compares these values with the provided two threshold values. The pixel is set to zero if gradient value is below the low threshold value and if it is above the high threshold value then pixel is set as an edge. In case if, the gradient value is in between the two threshold values by default that pixel is set to zero (regarded as non-edge) although there is a path from that pixel to the pixel having gradient value above the high threshold value. 


\subsection{Computed Tomography (CT)}

Computed Tomography (CT) scanning sometimes called Computed Axial Tomography (CAT) scanning [7], is a noninvasive medical test that helps physicians diagnose and treat medical conditions. CT scanning combines special x-ray equipment with sophisticated computers to produce multiple images or pictures of the inside of the body. These cross-sectional images of the area being studied can then be examined on a computer monitor, printed or transferred to a CD. CT scans of internal organs, bones, soft tissue and blood vessels provide greater clarity and reveal more details than regular x-ray exams. Using specialized equipment and expertise to create and interpret CT scans of the body, radiologists can more easily diagnose problems such as cancers, cardiovascular disease, infectious disease, appendicitis, trauma, and musculoskeletal disorders. Hence, the CT scan application is been widely used in medical domain. There are different segmentation methods proposed considering CT scan of different body organs (such as lung, liver, kidney, etc.) This section covers the different segmentation algorithm for CT scan images for protecting the distortion of diagnosis value.

The 2-D and 3-D segmentation of organs in medical application of image processing are classified into model based and nonmodel based approaches. Nonmodel based approaches depends on local information such as, texture, intensity, spatial correlation of 2-D organ image in consecutive slices, and the location of the organ in the abdominal area with respect to neighboring structures, e.g., spine and ribs [25 ]. Various segmentation algorithms are developed using nonmodel-based approach. This section first covers the different segmentation algorithm, which uses nonmodel-based approach. Susomboon et al. [26] presented texture features to perform region classification for extracting liver's soft tissue. Seo et al. [27] employed a multimodal threshold method based on piecewise linear interpolation that used spine location as a reference point. Forouzan et al. [28] introduced a multilayer threshold technique, in which by statistical analysis of the liver intensity it calculates the threshold value. Both these methods use the local information of the liver's relative position to the spine and ribs. Nonmodel-based methods for organ segmentation leads to inaccuracies due to variation in imaging condition, because of occurrence of tumor inside the organ and noise. Dependencies on prior information such as texture and image values could cause inaccuracies in segmentation process as feature could change from one patient to another. Moreover, most of these methods are parameter dependent and hence for the best performance it often needs to adjust the parameters from one CT volume to other. In recent years, model-based image segmentation algorithms developed for various medical applications. These methods aim to recover an organ based on statistical information. State-ofthe-art algorithms on model-based segmentation are based on active shape and appearance models [25, 29]. Model-based techniques provide more accurate and robust algorithm for segmenting the CT scan image. These techniques also deal with the missing image features via interpolation. The performance of these methods depends on the number and type of training data. Moreover, if the shape to be segmented lies too far from the model space, that might not be detected by many those better methods which does not implemented by statistical model-based approach.

Pan and Dawant [30] reported a geometrical-level set method for automatic segmentation of the liver in abdominal CT scans without relying on the prior knowledge of shape and size. Even if this method depends on a model-based technique, that outperforms threshold-based techniques, but it did not use prior knowledge of the liver shape. Lin et al. [31] presented the algorithm to perform segmentation of kidney, based on an adaptive region growing and an elliptical kidney region positioning that used spines as landmark. H. Badakhshannoory and P. Saeedi [32] incorporated a method for liver segmentation. Based on liver boundary edges to identify liver regions, nonrigid registration and a multilayer segmentation technique are combined in this approach. This method is does not affected by the diversity of existing liver shapes, as it does not 
rely on any shape model. Samuel et al. [33] has proposed the use of Ball-Algorithm for the segmentation of lungs. In this algorithm at the first stage, it applies the grey level thresholding to the CT images to segment the thorax from background and then the lungs from the thorax. Then in the next step to avoid loss of juxtapleural nodules, this method performs the rolling ball algorithm. Julian Ker [34] has presented the method of doing segmentation of lungs, which is named as TRACE method. Due to the possible presence of various disease processes, and the change of the anatomy with vertical position results in variation of size, shape, texture of lungs CT image of different patients. Therefore, the boundary between lung and surrounding tissues can vary from a smooth-edged, sharp-intensity transition to irregularly jagged edges with a less distinct intensity transition. The TRACE algorithm implemented with new perception of a nonapproximating technique for edge detection. Shiying et al. [35] have introduced a fully automatic method for identifying lungs in 3D pulmonary X-Ray CT images. The method follows three main steps:

- lung region is extracted from CT-Scan image by applying graylevel thresholding,

- by using a dynamic programming it identifies the anterior and posterior junction, to separate left and right lungs and

- to smooth the irregularities of boundary along the mediastinum nodule, it implements sequence of morphological operations

Ayman El-Baz et al. [36] have employed a fully automatic Computer-Assisted Diagnosis (CAD) system for lung cancer screening using chest spiral CT scans. This paper presents a system for detection of abnormalities, identification or classification of these abnormalities with respect to specific diagnosis, and provides the visualization of the results over computer networks. The process of detection of abnormalities, identification of these abnormalities can achieve by image analysis system for 3-D reconstruction of the lungs. Riccardo Boscolo et al. [37] proposed method that uses the novel segmentation technique that combines a knowledge based segmentation system with a sophisticated active contour model. This method performs robust segmentation of various anatomic structures. In this approach the user, need to provide initial contour placement, and the required parameter optimization automatically determined by the high-level process. Binsheng et al. [38] reported the algorithm, which used the method of selecting the threshold value by analyzing the histogram. This algorithm initially separates the lung parenchyma from the other anatomical structures from the CT images by using threshold value. By this algorithm structure in CT scan image with higher densities having some higher density nodules, can grouped into soft tissues and bones, leading to an incomplete extraction of lung mask. For having complete hollow free lung mask, morphological closing is applied in this approach. Hossein B. et al. [25] has introduced the model-based segmentation algorithm. In this approach instead of using model information to direct the segmentation algorithm for segmenting an organ of CT scan images, it uses this information to choose a segment with highest fidelity to the organ.

After completing with the segmentation of ROI, needs to proceed with medical image watermarking technique to provide security, authentication and privacy of this medical data. The next section of this paper provides the survey of different available medical image watermarking approaches.

\section{MEDiCAL IMAge WATERMARKING}

There has been fair amount of work done in the area of medical image processing. Before proceeding with survey of medical image processing, this section covers the foundation of digital watermarking, types of domain and performance measurement. The typical block diagram for medical image watermarking is given in figure 2 . 
Encoder $\mathrm{E}$ embeds the watermark $\mathrm{W}$ in medical image to provide security and authentication. Decoder D extracts the watermark from watermarked image. By comparing the extracted watermark with original watermark, one can affirm the tampering of medical image. According to watermark embedding process, watermarking techniques are classified into two different domains.

- Spatial domain: The spatial-domain watermark insertion manipulates image pixels. However, the spatial-domain watermark insertion is simple and easy to implement, it is weak versus various attacks and noise.

- Transform domain: The transform-domain watermark insertion is based on the transform coefficients of cover image. It is more robust against attacks. Discrete Cosine Transform (DCT), Discrete Wavelet Transform (DWT), and Discrete Fourier Transform (DFT) are three popular methods in transform domain.

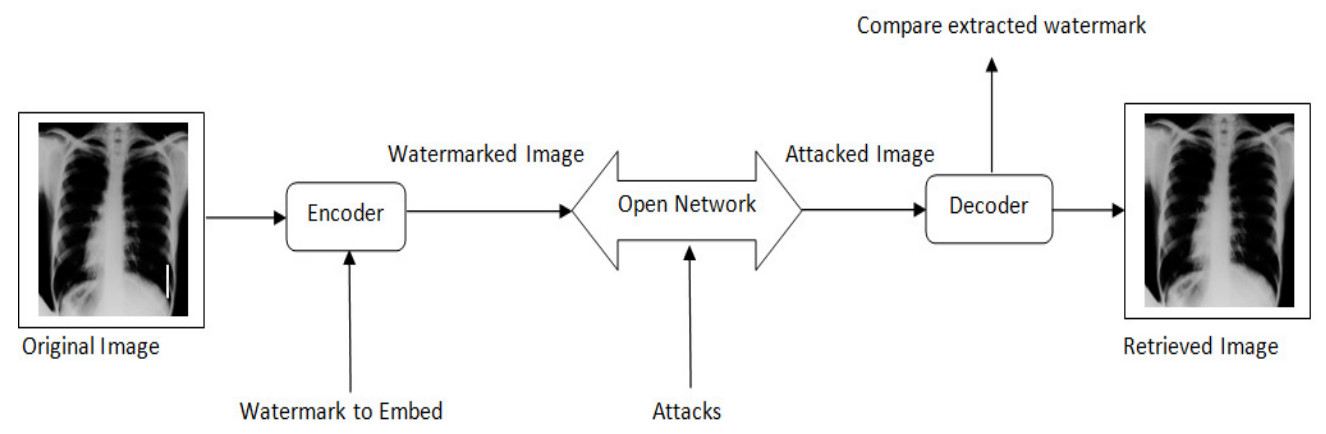

Fig. 2 Block Diagram of Medical Image Watermarking

To ensure the reliability and quality of the watermarked image, the performance of watermarking is calculated, which measured in terms of perceptibility. There are two method of calculating the performance measure.

- Mean Square Error (MSE): It is simplest function to measure the perceptual distance between watermarked and original image. MSE can be defined as:

$$
M S E=\frac{1}{n} \sum_{i}^{n}\left(I^{\prime}-I\right)^{2}
$$

Where, I is original image and I' is watermarked image.

- Peak Signal to Noise Ratio (PSNR): It is used to measure the similarity between images before and after watermarking.

$$
R S N R=10 \log _{10} \max I^{2}
$$

Where, max I is the peak value of original image.

Numbers of medical image watermarking schemes reported in this literature survey, to address the issues of medical information security, and authentication. 
Wakatani [39] presented a medical image watermarking, in order not to compromise with the diagnosis value, it avoids embedding watermark in the ROI. In this algorithm watermark to be embed is firstly compressed by progressive coding algorithm such as Embedded Zero Tree Wavelet (EZW). Embedding process is done by applying Discrete Wavelet Transform (DWT), for transforming the original image using Haar basis. Extraction of watermark is reverse of embedding process. The major drawback of this algorithm is ease of introducing copy attack on the non-watermarked area.

Yusuk Lim et al. [40] reported a web-based image authentication system, they used the CT scan images. This technique is mainly based on the principal of verifying the integrity and authenticity of medical images. In this approach, the watermark is preprocessed by using 7 most significant bit-planes except least significant bit (LSB) plane of cover medical image, as a input to the hash function. This hash function generates binary value of 0 or 1 using secrete key, which is then embedded in LSB bit of cover image to get watermarked image.

Rodriquez et al. [41] proposed a method in which it searches a suitable pixel to embed information using the spiral scan that, starts from the centroid of cover image. Then by obtaining the block with its center at the position of selected pixel, it checks the value of bit to embed. If bit value is 1 , then the embedding information is obtained by changing the luminance value of the central pixel by adding the gray-scale level mean of the block with luminance of the block. In addition, if bit value is 0 , then luminance value of the central pixel is changed by subtracting the luminance value of block from the gray-scale level mean of the block. While in extraction process, the position of marked pixel is obtained by spiral scan starting from centroid of the cover image. By checking the luminance value of the central pixel with the gray-scale level mean of the block, embedded bit is identified.

Giakoumaki et al. [1] presented a multiple watermarking method using wavelet-based scheme. The method provides solution to the number of medical data management and distribution issues, such as data confidentiality, archiving and retrieval, and record integrity. In this approach up to 4 level DWT is performed on medical image. The algorithm embeds multiple watermarks in different level. A robust watermark containing doctor's identification code is embedded in 4th level as here capacity is not the matter, only required is the robustness. In third level decomposition, the index watermark (e.g ICD-10 or ACR diagnostic codes) is embedded. The method embeds caption watermark holding patients personal information in second decomposition level. Moreover, a fragile watermark is embedded in forth-level decomposition. Extraction process is reverse of embedding process. Experimentation is done on ultrasounds medical images.

Hemin Golpira et al. [42] reported reversible blind watermarking. In this approach during embedding process, firstly by applying Integer Wavelet Transform (IDWT) image is decomposed into four subbands. By selecting two points, called thresholds, according to the capacity required for the watermark data, watermark is embedded. To get watermarked image Inverse Integer Wavelet Transform (IIDWT) is applied. In the extraction process, all of these stages are performed in reverse order to extract watermark as well as host image.

Nisar Ahmed Memonet et al. [43] presented fragile and robust watermarking technique for medical images. The method embeds two different watermarks, the robust watermark and fragile watermark in the medical images. The embedding process is start with separation of ROI and RONI from medical images. The robust watermark containing the electronic patient record (EPR), Doctor's identification code (DIC) and 1st bit-plane of ROI by extracting the LSBs is encrypted by using pseudo random sequence generated by user defined key. Then this resultant watermark is embedded in high frequency coefficient of IWT in RONI part of medical data. The proposed method generates fragile watermark by creating the binary image in tiled fashion and 
then this fragile watermark is cropped off by the same size as the ROI. The algorithm embeds this fragile watermark into spatial domain of ROI part of medical image. The extraction process is reverse of embedding process.

\section{CONCLUSION AND FUTURE WORK}

There exists various medical image watermarking algorithms and different existing segmentation algorithms to segment ROI. The segmentation algorithms vary for the types of medical images such as MRI, CT, US, etc. The current study work can further be extended to develop a GUI tool based approach for separating the ROI. Additionally, a new technique of separating ROI form the original image that will be applicable for all type of medical images can be evolved. Separated ROI can be stored with xmin, xmax, ymin, and ymax value so that at the end of embedding process before transmitting watermarked image, the segmented ROI can be attached with watermarked image. Any medical image watermarking approach will be suitable, if we segment the ROI from medical image with the four values, then embedding of watermark can be done on whole medical image. Therefore, space for watermark embedding will be more in such cases.

\section{ACKNOWLEDGEMENTS}

We take this opportunity to express my hearty thanks to all those who helped me in the completion of my survey paper. We are very grateful to the authors of various articles on the internet, for helping us become aware of the research currently ongoing in this field.

\section{REFERENCES}

[1] Giakoumaki, Sotiris Pavlopoulos, and Dimitris Koutsouris, (Oct. 2006) "Multiple Image Watermarking Applied to Health Information Management", IEEE Trans. on information technology in biomedicine, vol. 10, no. 4

[2] Imen Fourati Kallel, Mohamed Kallel, Mohamed Salim BOUHLEL, (2006 ) "A Secure fragile Watermarking Algorithm for medical Image Authentication in the DCT Domain", IEEE

[3] M.S.Bouhlel, (Mars 2002) "Conception d'une banque d'images medicales sur INTERNET", 3eme Rencontres Institutionnelles: Rhones Alpes/ Tunisie (RIRAT'02). Tozeur, Tunisie, 21-22

[4] Preeti Aggarwal, Renu Vig, Sonali Bhadoria, and C.G.Dethe , (September 2011) "Role of Segmentation in Medical Imaging: A Comparative Study", International Journal of Computer Applications (0975 - 8887), Volume 29- No.1

[5] Pradeep Singh; Sukhwinder Singh, Gurjinder Kaur, (2008) "A Study of Gaps in CBMIR using Different Methods and Prospective, Proceedings of world academy of science, engineering and technology", volume 36, ISSN 2070-3740, pp. 492-496.

[6] Zhen Ma, Joao Manuel, R. S. Tavares, R. M. Natal Jorge, (2009) "A review on the current segmentation algorithms for medical images", 1st International Conference on Imaging Theory and Applications (IMAGAPP), Lisboa, Portugal, INSTICC Press, pp. 135-140.

[7] Nisar Ahmed Memon, Anwar Majid Mirza, and S.A.M. Gilani, (2006) "Segmentation of Lungs from CT Scan Images for Early Diagnosis of Lung Cancer", World Academy of Science, Engineering and Technology 20.

[8] Parmida Moradi Birgani, Meghdad Ashtiyani, and Saeed Asadi, "MRI Segmentation Using Fuzzy Cmeans Clustering Algorithm Basis Neural Network"

[9] M. M. Khalighi, H.S.Zadeh, C. Lucas, (Feb 2002) "Unsupervised MRI segmentation with spatial connectivity", 23-28, San Diego, CA. "in press"

[10] L. Jiang, W. Yang, (Dec 2003) "A modified fuzzy c-means algorithm for segmentation of MR Images" Proc. VII ${ }^{\text {th }}$ Digital Image Computing: Techniques and Applications. , 10-12, Sydney. "in pres"

[11] M.Y. Siyal, L. Yu, (2005) "An intelligent modified fuzzy c-means based algorithm for bias estimation and segmentation of brain MRI Pattern Recognition Letters”, pp. 2052-2062 
[12] Moslem Taghizadeh, Mahboobeh Hajipoor, 2011 “A Hybrid Algorithm for Segmentation of MRI Images Based on Edge Detection”, 2011 International Conference of Soft Computing and Pattern Recognition (SoCPaR)

[13] M.Sezgin, (2004) "Survey over image thresholding techniques and quantitative performance evaluation", Journal of Electronic Imaging 13(1), pp.146-165

[14] Yavuz, Z., (2010) "Comparing 2D matched filter response and Gabor filter methods for vessel segmentation in retinal images", IEEE Trans. Electrical, Electronics and Computer Engineering (ELECO), vol.8, no.6,pp.648-652

[15] Dong-Chul Park, (2010) "Intuitive Fuzzy C-Means Algorithm for MRI Segmentation”, 978-1-42445943-8/10/, IEEE

[16] J.Bezdek,( 1981 ) "Pattern recognition with fuzzy objective function algorithms" Plenum, New York,

[17] P. Wang and H. Wang., (2008) "A Modified FCM Algorithm for MRI B rain Image Segmentation", Proc. Fut. Biomed. Info. Eng., 26-29

[18] R. Krishnapuram and J. Keller, (1993) “A possibilistic approach to clustering”, IEEE Trans. Fuzzy Syst., 1(2) : 98-110,

[19] N. Pal, K. Pal, and J. Bezdek, (2005) “A Possibilistic Fuzzy c-Means Clustering Algorithm”, IEEE Trans. Fuzzy Sys., 13(4), pp. 517-530,

[20] Talib Hussein R., (2009) "Automatic Extracted Object Technique for Contrast Enhancement Medical Images”, IJCCCE, VOL.9, NO.1,

[21] Civicioglu P., (2003) "CCII based analog circuit for the edge detection of MRI images", IEEE Trans. Micro-NanoMechatronics and Human Science, vol.1, no.6, pp.341-344,

[22] Sachin G Bagul, (2011) “Comparison of SUSAN and Sobel Edge Detection in MRI Images for Feature Extraction”, IJCA Journal, VOL.1, NO.1, USA.

[23] J. F. Canny, (1986), "A computational approach to edge detection”, IEEE Trans. Pattern Analysis and Machine Intelligence, vol.8, no.6, pp.679-698

[24] D. Marr and E.C. Hildreth, (1980) "Theory of edge detection”, Proc. Roy. Soc. London, B207, pp.187-217

[25] Hossein Badakhshannoory and Parvaneh Saeedi, (September 2011) "A Model-Based Validation Scheme for Organ Segmentation in CT Scan Volumes", IEEE Trans. on biomedical information, vol. 58 , no. 9 ,

[26] R. Susomboon, D. Raicu, and J. Furst, (2007) “A hybrid approach for liver segmentation”, in Proc. 3D Segment. Clin.-MICCAI Grand Challenge

[27] K. Seo, L. C. Ludeman, S. Park, and J. Park, (2005) "Efficient liver segmentation based on the spine", Adv. Inf. Syst., vol. 3261, pp. 400-409

[28] A. H. Forouzan, R. A. Zoroofi, M. Hori, and Y. Sato, (2009) "Liver segmentation by intensity analysis and anatomical information in multislice CT images”, in Proc. Liver Segment. Intensity Anal Anatomical Inf. Multi-Slice CT Images, vol. 4, pp. 287-297

[29] T. F. Cootes, C. J. Taylor, and D. H. Cooper, (2001) "Statistical models of appearance for medical image analysis and computer vision," Proc. SPIE, vol. 4322, pp. 236-248

[30] S. Pan and B. M. Dawant, (2001) "Automatic 3D segmentation of the liver from abdominal CT images: A level-set approach," Proc. SPIE, vol. 4322, pp. 128-138

[31] D. T. Lin, C. C. Lei, and S. W. Hung, (Jan 2006) "Computer-aided kidney segmentation on abdominal CT images”, IEEE Trans. Inf. Technol. Biomed.,vol. 10, no. 1, pp. 59-65

[32] H. Badakhshannoory and P. Saeedi, (2010) "Liver segmentation based on de-formable registration and multilayer segmentation," in Proc. IEEE Int. Conf. Image Process., pp. 2549-2552

[33] Samuel G. Armato III, Maryellen L. Giger and Catherine J. Moran, (1999) "Computerized Detection of Pulmonary Nodules on CT Scans", RadioGraphics, vol. 19, pp. 1303-1311

[34] Julian Kerr, (May 2000) “The TRACE method for Segmentation of Lungs from Chest CT images by Deterministic Edge Linking”, University of New South Wales, Department of Artificial Intelligence, Australia

[35] Shiying Hu, Eric A.Huffman, and Joseph M. Reinhardt, (June 2001) “Automatic Lung Segementation for Accurate Quantitiation of Volumetric X-Ray CT images”, IEEE Transactions on Medical Imaging, vol. 20, No. 6

[36] Ayman El-Baz, Aly A. Farag, Robert Falk, and Renato La Rocc, (Jan 2002) " Detection, Visualization, and Identification of Lung Abnormalities in Chest Spiral CT Scans: Phase 1", International Conference on Biomedical Engineering, Cairo, Egypt

[37] Riccardo Boscolo, Mathew S. Brown, Michael F. McNitt-Gray, (2002) "Medical Image Segmentation with Knowledge-guided Robust Active Contours”, Radiographics, vol. 22, pp. 437-448 
[38] Binsheng Zhao, Gordon Gamsu, Michelle S. Ginsberg, (2003) "Automatic detection of small lung nodules on CT utilizing a local density maximum algorithm”, Journal of Applied Clinical Medical Physics, vol. 4, No. 3

[39] A. Wakatani, (Jan 2002) "Digital Watermarking for ROI Medical Images by Using Compressed Signature Image", Proceedings of the 35th International Conference on System Sciences,

[40] Yusuk Lim, Changsheng Xu, and David Dagan Feng, "Web based Image Authentication Using Invisible Fragile Watermark", Pan-Sydney Area Workshop on Visual Information Processing (VIP2001), Sydney, Australia

[41] C.R. Rodriguez, F. Uribe Claudia, T. Blas Gershom De J, (2007) "Data Hiding Scheme for Medical Images", IEEE $17^{\text {th }}$ International Conference on Electronics, communications and computers

[42] Hemin Golpira and Habibollah Danyali, (2009)"Reversible Blind Watermarking for Medical Images Based on Wavelet Histogram Shifting", IEEE

[43] Nisar Ahmed Memon, S.A.M. Gilani, and Shams Qayoom, (2009) "Multiple Watermarking of Medical Images for Content Authentication and Recovery", IEEE

\section{Authors}

1) Ms. Sonika Rathi is pursuing her M. Tech in computer Engineering from College of Engineering Pune, Maharashtra. She is graduated from S wami Ramanand Tirth Marathwada University, Nanded in 2009. Her research interest are watermarking, data mining and medical informatics.

2) Mrs. Vandana Shridhar Inamdar is Assistant Professor, at Department of Computer Engineering \& IT, College of Engineering Pune, Maharashtra. She is having more than 20 years experience in teaching along with 2 years in dustry experience. She has registered for Ph.d in Pune university. Her Topic of research is- "Biometric watermarking for digital media". She has completed her M.E. Electronics from Pune University, 1997. she is graduated from Shivaji University, 1989.

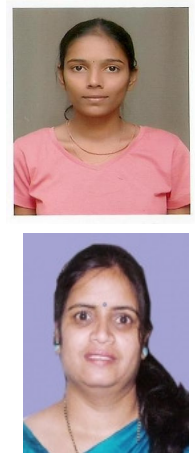

